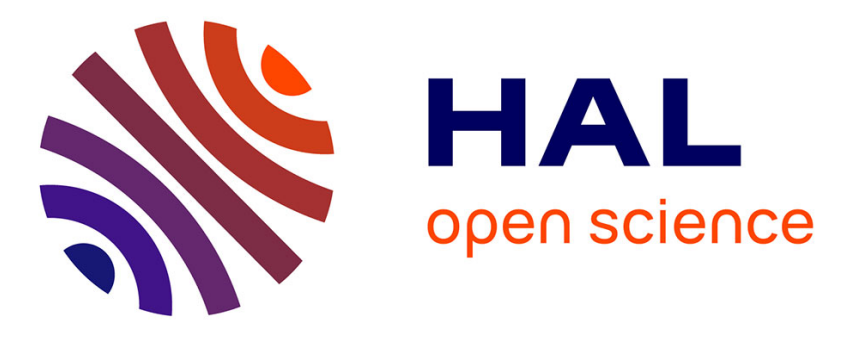

\title{
Fluid dynamics of rivulet flow between plates
}

W. Drenckhan, H. Ritacco, Arnaud Saint-Jalmes, A. Saugey, P. Mcguinness, A. van Der Net, Dominique Langevin, Denis Weaire

\section{To cite this version:}

W. Drenckhan, H. Ritacco, Arnaud Saint-Jalmes, A. Saugey, P. Mcguinness, et al.. Fluid dynamics of rivulet flow between plates. Physics of Fluids, 2007, 19, pp.102101. 10.1063/1.2757153 . hal00905300

\section{HAL Id: hal-00905300 https://hal.science/hal-00905300}

Submitted on 18 Nov 2013

HAL is a multi-disciplinary open access archive for the deposit and dissemination of scientific research documents, whether they are published or not. The documents may come from teaching and research institutions in France or abroad, or from public or private research centers.
L'archive ouverte pluridisciplinaire HAL, est destinée au dépôt et à la diffusion de documents scientifiques de niveau recherche, publiés ou non, émanant des établissements d'enseignement et de recherche français ou étrangers, des laboratoires publics ou privés. 


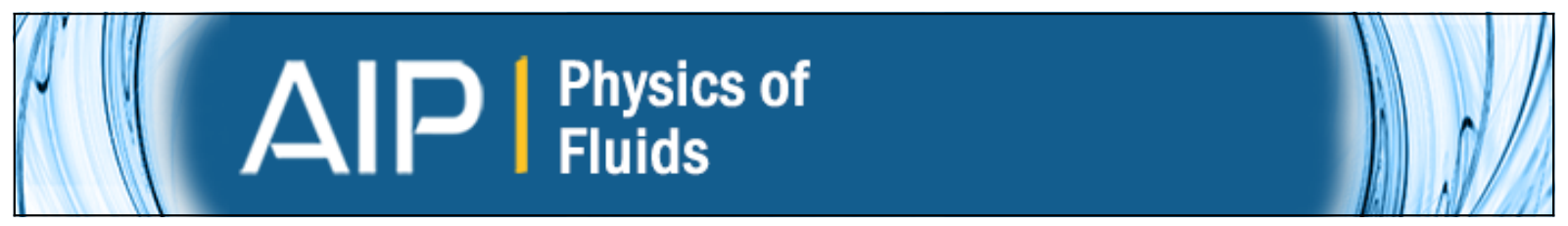

\section{Fluid dynamics of rivulet flow between plates}

W. Drenckhan, H. Ritacco, A. Saint-Jalmes, A. Saugey, P. McGuinness, A. van der Net, D. Langevin, and D. Weaire

Citation: Physics of Fluids (1994-present) 19, 102101 (2007); doi: 10.1063/1.2757153

View online: http://dx.doi.org/10.1063/1.2757153

View Table of Contents: http://scitation.aip.org/content/aip/journal/pof2/19/10?ver=pdfcov

Published by the AIP Publishing

\section{AlP Re-register for Table of Content Alerts}




\title{
Fluid dynamics of rivulet flow between plates
}

\author{
W. Drenckhan \\ School of Physics, Trinity College, Dublin, Ireland \\ H. Ritacco and A. Saint-Jalmes \\ Laboratoire de Physique des Solides, Université Paris-Sud, Orsay, France \\ A. Saugey, P. McGuinness, and A. van der Net \\ School of Physics, Trinity College, Dublin, Ireland \\ D. Langevin \\ Laboratoire de Physique des Solides, Université Paris-Sud, Orsay, France \\ D. Weaire \\ School of Physics, Trinity College, Dublin, Ireland
}

(Received 8 March 2007; accepted 16 May 2007; published online 3 October 2007)

\begin{abstract}
We present computational and experimental investigations into the fluid dynamics of a narrow stream of surfactant solutions, which descends under gravity between two narrowly spaced, vertical glass plates. Such a "rivulet" is bounded by two liquid/solid and two mobile liquid/gas interfaces, posing fluid dynamic problems of direct relevance to local fluid flow in liquid foams and recently reported meandering phenomena. The rivulet presents a system in which the coupling between the bulk flow and the rheological properties of the gas/liquid interface can be systematically investigated. In particular, it carries the promise of providing an alternative measuring technique for interfacial shear viscosities. We present finite element simulations in conjunction with experiments in order to describe the relationship between the rivulet geometry, the flow field, and the interfacial shear viscosities. We also report on the role of the boundary condition between the liquid-carrying channels (surface Plateau borders) and the thin soap film, which spans the two plates at low flow rates. (c) 2007 American Institute of Physics. [DOI: 10.1063/1.2757153]
\end{abstract}

\section{INTRODUCTION}

Recent years have seen the development of a range of analytical and numerical methods to successfully describe the flow of (Newtonian) liquids through channels with boundaries of general shape and properties. In particular, one can now deal with boundaries which are liquid/liquid or liquid/gas interfaces, as found in foams and emulsions, which respond to flow by adjusting their shape. In the case considered here, there is a dramatic effect of this kind, a strikingly regular meandering instability. ${ }^{1}$

There is another kind of mobility to be considered, namely that of flow in the channel surfaces itself, which provides an intricate coupling between surface and bulk flow, often complicated by the fact that many of these systems are stabilized by surface active species. Their interaction provides the channel surfaces not only with a shear and dilational viscosity but also with a shear and dilational elasticity, ${ }^{2}$ which (to a varying degree) have proven to be essential ingredients in the theoretical description of the flow and stability of such systems. ${ }^{2,3}$

In particular the interfacial shear viscosity $\mu_{s}$ has been established as a crucial parameter in the description of liquid flow through foams. It is generally expressed in terms of the dimensionless mobility parameter

$$
M=\frac{\mu_{l} L}{\mu_{s}},
$$

where $\mu_{l}$ is the liquid viscosity and $L$ a typical length scale of the channel. The mobility $M$ was originally introduced to the fluid dynamics of foams by Leonard and Lemlich ${ }^{4}$ and has since been successfully included in the description of a number of related problems. ${ }^{5-9}$

For $M \ll 1$ one may speak of (tangentially) immobile surfaces, which impose a no-slip boundary condition and therefore lead to Poiseuille-type flow, as in the case of solid pipes. For $M \gg 1$ the surface is (tangentially) mobile and does not impose any tangential stresses on the bulk, leading to plug-like flow. In the intermediate case, when $M$ is of the order of unity, surface and bulk are coupled in a less obvious way.

In many cases the channels are partially bounded by solid surfaces or held in place by thin films, which impose additional stresses. The latter have since Leonard and Lemlich $^{4}$ been assumed to provide a no-slip condition. Computational and experimental investigations into the detailed interplay between these boundary conditions (which also influence the channel geometry) and the mobility parameter $M$ have been provided in particular by Nguyen ${ }^{5}$ and Koehler et al. ${ }^{6,8,9}$

In this article, we extend previous findings by introducing investigations into the flow through a very simplified system (see Sec. II), representative of the local flow in a foam: a "rivulet", which-at sufficiently low flow ratesconsists of only one single soap film, which spans two narrowly spaced glass plates with a liquid pocket (surface Plateau border) at either side. In addition to the simple and well defined geometry, it provides access to a regime at higher flow rates, in which the thin film is replaced by a liquid 


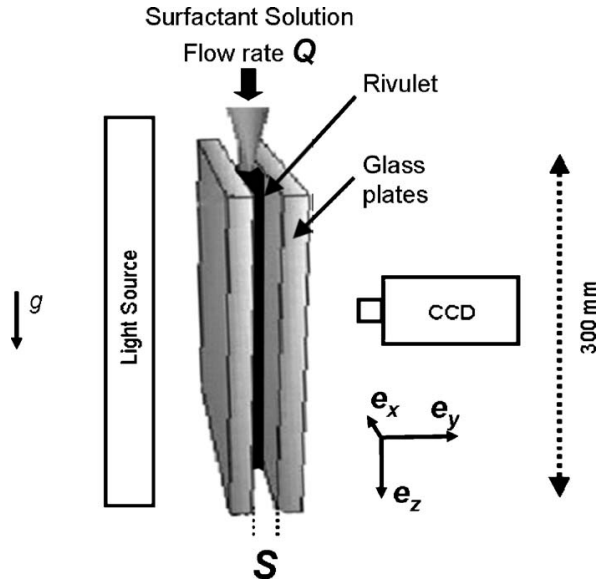

FIG. 1. Sketch of the setup: surfactant solution is injected at constant flow rate $Q$ between the two narrowly spaced, vertical glass plates. The liquid descends under gravity and forms a rivulet, which is straight and vertical at sufficiently low flow rates.

bridge that spans the plates. As far as we are aware, this regime has not been studied previously; certainly not in surfactant systems.

In both regimes, which are described in more detail in Sec. II, we computationally relate the flow field to the rivulet geometry and the interfacial mobility $M$ (Sec. IV), using finite element techniques. Our results are compared with experiments in Sec. V, which are in good agreement. Contrary to what is observed in foams, we find that the rivulet system seems to be best described by assuming a full-slip flow condition where the thin film is attached to the surface Plateau borders.

A full understanding of the rivulet flow carries the promise of providing a novel, and straightforward, method to measure interfacial shear viscosities. Major advantages would lie in its translational symmetry and the fact that measurements are taken in the steady state. This allows us to focus on the interfacial shear viscosity as the sole interfacial parameter. An additional advantage is given by the fact that the experiment provides access to two different flow regimes, making possible the elimination or emphasis of specific effects by contrasting the flow properties in both regimes.

Our investigations are also intended to feed into an understanding of the stability of the rivulet system, which has been shown to display perfectly regular meanders in various regimes above a critical flow rate with a significant dependence on the surfactants used to stabilize the film. ${ }^{1}$ To this end, we present computational results on the maximum velocities encountered in the rivulet system in Appendix B.

\section{GENERAL DESCRIPTION OF THE RIVULET SYSTEM}

As sketched in Fig. 1, surfactant solution is injected at a constant flow rate $Q$ between two narrowly spaced, parallel and vertical glass plates. The liquid descends under gravity, forming the so-called "rivulet" which spans the plates. Here we are concerned with the stable rivulet, which is straight and vertical at sufficiently low flow rates. ${ }^{1}$

As shown in Fig. 2, such a rivulet is bounded by two liquid/solid and two liquid/gas surfaces, $\partial \Omega_{l s}$ and $\partial \Omega_{l g}$, of which the latter adjust to the flow. Sufficiently far below the nozzle (beyond the "entry length"), the flow is equilibrated and the rivulet cross section remains constant, i.e., it has translational symmetry along the vertical $z$-axis. The width $W$ of the rivulet is a function of the flow rate $Q$, and this article is largely concerned with relating these two quantities as a function of the rheological properties of the gas/liquid interface.

Depending on the flow rate, the rivulet has two significantly different types of cross section (Fig. 2). At low flow rates (left of Fig. 2), a thin soap film is stabilized by the surfactants ${ }^{10}$ and attached to the plates by so-called surface Plateau borders which carry most of the liquid. Assuming the liquid to be perfectly wetting (which is an excellent approximation for surfactant solutions on glass), the cross section of the Plateau borders is bounded by two quarter-arcs of circles. Their radius of curvature $R$ is given by the Laplace law, which balances the liquid/gas interfacial tension $\gamma$ with the pressure difference $\Delta p$ between the gas and the liquid

$$
\Delta p=\frac{\gamma}{R}
$$

The width $W$ of this rivulet is given by $W=2 R+F . F$ is the thickness of the film, which is generally very small $\left[F=O\left(10^{-3} R\right)\right]$, so that $W \approx 2 R$.

With increasing flow rate $Q$, the Plateau borders swell without changing their shape until their radius of curvature $R$
Regime I

\section{Surface Plateau borders} connected by a thin film

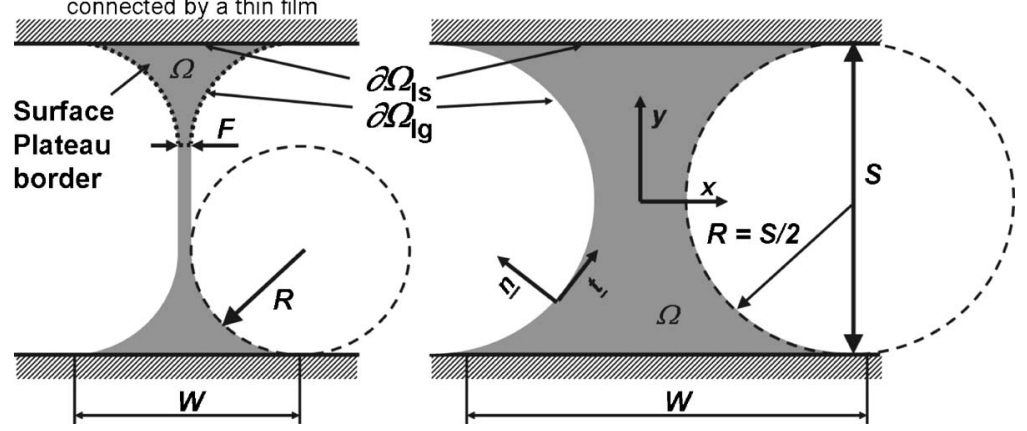

FIG. 2. The rivulet has two different cross sections depending on the flow rate $Q$. At low flow rates (left image) two surface Plateau borders (dotted line) are connected by a thin film. These merge to form a liquid bridge at sufficiently high flow rates (right image). The gas/liquid boundaries are described by arcs of circles of radius $R$. The liquid domain is labelled $\Omega$, while the liquid/solid and liquid/gas boundaries are labelled $\partial \Omega_{l s}$ and $\partial \Omega_{l g}$, respectively. 
is half of the plate spacing $S$, i.e., $W=2 R=S$ (assuming $F \ll R)$. Beyond this point, the thin film disappears and the Plateau borders merge to form a liquid bridge, as shown on the right-hand side of Fig. 2. Now the gas/liquid interfaces form semicircles with fixed radius of curvature $R=S / 2$ [given by Eq. (2)], which are pushed outwards with further increase of flow rate, leading to a different scaling behavior than in the Plateau border regime.

The point at which the Plateau borders merge $(W=S)$ marks the transition between two different flow regimes, which is expected to be reflected in the dependence of the rivulet width $W$ on the flow rate $Q$.

In both regimes we choose the radius of curvature $R$ of the gas/liquid interface as the representative length scale in the definition of the dimensionless mobility parameter

$$
M=\frac{\mu_{l} R}{\mu_{s}} .
$$

This simplifies the formalism after nondimensionalization, as shown in Sec. IV. ${ }^{4}$ Thanks to the scale-invariance of the surface Plateau border geometry, the flow field in the low flow rate regime is uniquely characterized by the mobility parameter $M$, which contains the rivulet width $W=2 R$ (Sec. IV C). In the liquid bridge regime, $W$ has to be taken into account explicitly in addition to the mobility parameter $M$ (Sec. IV D).

We have chosen to present the theory and computational results in Sec. IV in nondimensional form. This not only allows straightforward comparison with previous work, ${ }^{4-6,8,9}$ but also highlights the key parameters and their relationships. For historical reasons we have also chosen to present $Q$ as a function of $W$, because in the simulations $W$ is set to find the corresponding $Q$ (while it is the opposite in experiments).

For comparison with experiments in Sec. V we redimensionalized the computational results using the respective physical values for each set of data. Within this redimensionalization, the reader needs to be aware that even though the surface viscosity $\mu_{s}$ of the gas/liquid interface remains constant in each experiment, $M$ is constant only in the liquid bridge regime on account of the variation of $R$.

\section{ROLE OF THE THIN FILM}

The influence of the thin film which is formed between the plates in the surface Plateau border regime (Fig. 2) deserves special attention. The amount of liquid contained or flowing in this film is generally negligibly small, particularly in systems of high surface viscosities. ${ }^{8}$ For the analysis of the flow through this rivulet geometry (Sec. IV C) it is therefore sufficient to consider the flow through the surface Plateau borders only, as indicated by the dotted line in Fig. 2. What is not negligible, however, is the boundary condition this film provides on the Plateau border flow. Building on work by Leonard and Lemlich, ${ }^{4}$ nearly all previous literature on foam physics evokes a no-slip condition at this boundary. ${ }^{3,5,8}$ This assumption has been confirmed experimentally for a range of dry foams by Koehler et al. ${ }^{9}$ Its origin is generally attributed to recirculation motion within the film as a result of Marangoni stresses, ${ }^{11}$ but is still not well understood.

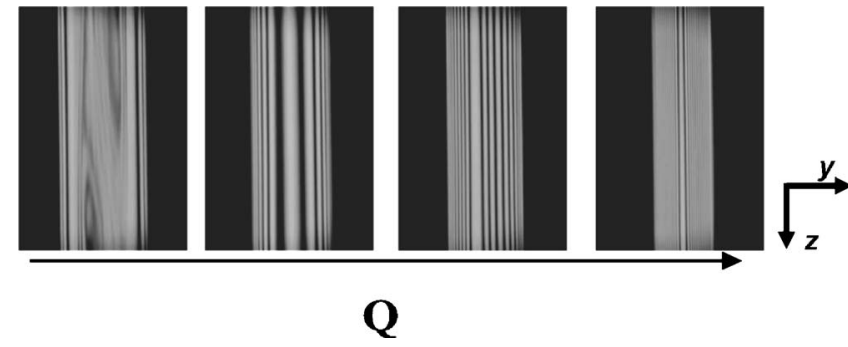

FIG. 3. Monochromatic light interference at the thin film in the Plateau border regime (Fig. 2), viewed from the side (x-direction in Fig. 1). Complex swirling motion is only seen at very low flow rates $Q$, while a laminar flow is found over the whole range of $Q$ applied for the measurements presented in this article.

Our rivulet system, however, differs significantly from that of ordinary foams. Here the film is very long $(\approx 30 \mathrm{~cm})$ and narrow $(<1 \mathrm{~mm})$, and also the flow rates are much higher than those commonly encountered in bulk foams.

In order to obtain information about the flow in the thin film, we have looked at the rivulet from the side, using the interference of monochromatic light (sodium, $\lambda=589 \mathrm{~nm}$ ) reflected off the two interfaces of the film. The resulting interference pattern provides information on thickness variations in the film.

A typical example $(S=2 \mathrm{~mm})$ of our observations for increasing flow rate is shown in Fig. 3. The black boundaries correspond to the Plateau borders, while the bright central part corresponds to the thin film. Only at very low flow rates (left picture in Fig. 3), which are below the measurable threshold of our equipment, do we see the swirling motion often described for larger soap films. For all flow rates within the measurable range, the thickness variations of the film becomes completely steady. Since this stable pattern is observed all the way into the Plateau border, we take this as an indication that the flow in the film is fully laminar and downwards.

We therefore assume that the film is simply carried along by the flow in the Plateau border. We also assume that the film is effectively weightless, i.e., that the stresses it exerts on the Plateau border are negligibly small. This leads to the assumption of a full-slip condition at the contact line between film and Plateau border, which we implement in our simulations (Sec. IV C). For comparison, we contrast these results in some places with those obtained with a no-slip condition (Sec. IV C 2).

Future research should settle such questions quantitatively by using velocimetry (or equivalent) techniques.

Regarding the film thickness, the interference patterns show that it always has a minimum in the center (Fig. 3), and that its thickness $F$ remains below a few $\mu \mathrm{m}$. The ratio $F / R$ thus remains smaller than 0.01 , and the flow rate in the film is therefore negligible (as long as $M<100$ ). This was shown by Koehler et al., ${ }^{8}$ who compared the magnitude of liquid flow through films and Plateau borders for different surface shear viscosities.

In the experiments, there is also a thin liquid film wetting the plates. We neglect this here, as we consider its influence on the overall flow negligible. 


\section{THEORY AND SIMULATION}

\section{A. General theory}

A theoretical description needs to relate the bulk flow through the rivulet to the conditions on the boundary. Because of their different geometries and scaling behavior, the two flow regimes (Fig. 2) are treated separately in Secs. IV C and IV D. In this section we introduce the general principles, upon which our analysis is based.

We assume that Reynolds numbers are sufficiently small such that the flow can be taken to be laminar and uniaxial. Taking also into account the translational symmetry, the problem can be reduced to two dimensions $(x, y)$ by considering the flow through the rivulet cross section $\Omega$ (Fig. 2) only.

The corresponding two-dimensional velocity field $u_{z}(x, y)=u(x, y)$ is related to the flow rate $Q$ as

$$
Q=\int u(x, y) d \Omega,
$$

which can be measured in the experiment.

In order to stabilize the thin film at low flow rates (lefthand side of Fig. 2), only a small amount of surface active species is needed (concentration $<1 \%$ ). The bulk properties therefore remain unchanged and are assumed to be Newtonian with bulk viscosity $\mu_{l}$ and density $\rho$. In the steady state, gravitational forces $\rho g$ are balanced by bulk dissipation

$$
\mu_{l} \Delta u(x, y)=\mu_{l}\left[\frac{\partial^{2} u(x, y)}{\partial x^{2}}+\frac{\partial^{2} u(x, y)}{\partial y^{2}}\right]=\rho g \quad \text { on } \Omega .
$$

The mathematical description of the problem is completed by establishing the conditions on the boundaries $\partial \Omega$. The liquid/solid boundaries provide a no-slip condition

$$
u(x, y)=0 \quad \text { on } \partial \Omega_{l s} .
$$

The gas/liquid interface $\partial \Omega_{l g}$ has a finite surface shear viscosity $\mu_{s}$ whose magnitude depends on the properties of the surface active species. It exerts tangential stresses on the bulk flow ${ }^{2,4-6}$ which must be balanced by the bulk stresses. Hence, ${ }^{2}$

$$
\mu_{l}(\underline{n} \cdot \nabla) u(x, y)=\mu_{s}(\underline{t} \cdot \nabla)^{2} u(x, y) \quad \text { on } \partial \Omega_{l g}
$$

or

$$
\begin{aligned}
& \mu_{l}\left[n_{x} \frac{\partial}{\partial x}+n_{y} \frac{\partial}{\partial y}\right] u(x, y) \\
& \quad=\mu_{s}\left[t_{x} \frac{\partial}{\partial x}+t_{y} \frac{\partial}{\partial y}\right]^{2} u(x, y) \quad \text { on } \partial \Omega_{l g},
\end{aligned}
$$

where $\underline{n}$ and $\underline{t}$ are the unit normal and tangential vectors on the interface, respectively. We assume that the shear elasticity is negligible, as usual in surfactant systems. ${ }^{2}$ The dilational elasticity and viscosity of the interface do not come into play here, on account of the translational symmetry.

\section{B. Computational approach}

Equations (5), (6), and (8) constitute the system of partial differential equations (PDEs) of the problem, which we solve using finite element techniques (COMSOL in combination with MATLAB).

For stability reasons and to be able to apply a PDE on the gas/liquid boundary, we rewrite Eq. (5) in its weak form, ${ }^{12}$ which is equivalent to applying the variation principle to the problem. For this we multiply Eq. (5) by a test function $u_{\mathrm{test}}(x, y)$ and then integrate over $d \Omega$,

$$
-\int_{\Omega} \mu_{l} u_{\mathrm{test}} \Delta u+\int_{\Omega} \mu_{l} \rho g u_{\mathrm{test}}=0 .
$$

Integrating the left-hand side by parts, transforming the integral using Green's theorem, and using Eq. (7) one obtains

$$
\begin{aligned}
0= & \int_{\Omega}\left(\mu_{l} \nabla u_{\text {test }} \cdot \nabla u+\rho g u_{\text {test }}\right)-\int_{\partial \Omega} \mu_{s} u_{\text {test }}(\underline{n} \cdot \nabla) u \\
= & \int_{\Omega}\left(\mu_{l} \nabla u_{\text {test }} \cdot \nabla u+\rho g u_{\text {test }}\right)-\int_{\partial \Omega} \mu_{s} u_{\text {test }}(\underline{t} \cdot \nabla)^{2} u \\
= & \int_{\Omega}\left(\mu_{l} \nabla u_{\text {test }} \cdot \nabla u+\rho g u_{\text {test }}\right) \\
& +\int_{\partial \Omega} \mu_{s}(\underline{t} \cdot \nabla) u_{\text {test }}(\underline{t} \cdot \nabla) u-\left[\mu_{s} u_{\text {test }}(\underline{t} \cdot \nabla) u\right]_{\partial^{2} \Omega}
\end{aligned}
$$

which combines the bulk $(\Omega)$, boundary $(\partial \Omega)$, and point $\left(\partial^{2} \Omega\right)$ conditions in one equation. The last term is the difference between the expression in the brackets at the extremities of the boundary (the sign being given by the direction one chooses for the tangent $t$ ). In the simulation these values are implemented through the constraints. The implementation in COMSOL is provided in Appendix A.

The simulations are conducted with fixed geometries, in which the velocity field (and therefore flow rate) is the adjusting parameter. The Dirichlet condition, which ensures that liquid does not penetrate the rivulet boundaries, is implicitly implemented through the uniaxial flow assumption.

In the Plateau border regime, the flow condition between the Plateau border and liquid film deserves some special attention and is therefore discussed in more detail in Secs. III and IV C.

\section{The surface Plateau border regime \\ 1. Nondimensionalization}

We consider the surface Plateau borders as separate entities (dotted line in Fig. 2) with radius of curvature $R$, which adjust to the flow rate $Q$ without changing their shape. We use the reduced spatial variables $x^{*}$ and $y^{*}$, with

$$
x=R x^{*} \quad \text { and } \quad y=R y^{*} .
$$

Introducing the reduced velocity $u^{*}\left(x^{*}, y^{*}\right)$ according to 


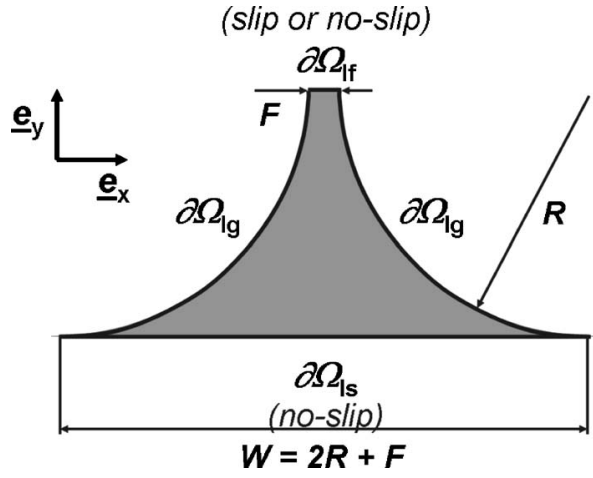

FIG. 4. Labelling used for surface Plateau border (see also the left-hand side of Fig. 2). We apply a full-slip condition at the boundary between the Plateau border and film $\partial \Omega_{l f}$.

$$
u^{*}\left(x^{*}, y^{*}\right)=\frac{\mu_{l}}{\rho g R^{2}} u(x, y),
$$

and the mobility parameter $M$, as defined in Eq. (3), Eqs. (5)-(7) simplify to

$$
\begin{aligned}
& \Delta^{*} u^{*}=1 \quad \text { on } \Omega, \\
& \left(\underline{n} \cdot \nabla^{*}\right) u^{*}=\frac{1}{M}\left(\underline{t} \cdot \nabla^{*}\right)^{2} u^{*} \quad \text { on } \partial \Omega_{l g}, \\
& u^{*}=0 \quad \text { on } \partial \Omega_{l s} .
\end{aligned}
$$

Having comparison with experiments in mind, we present our results in terms of the flow rate $Q$, which is expressed in its reduced form as

$$
Q^{*}=\int u^{*}\left(x^{*}, y^{*}\right) d \Omega=\frac{\mu_{l}}{\rho g R^{4}} Q .
$$

For the reasons stated in Sec. II we assume a full-slip condition where the Plateau border meets the film, which implies zero shear-rate (Fig. 4)

$$
\frac{\partial u^{*}}{\partial x}=0 \quad \text { on } \partial \Omega_{l f} \text {. }
$$

Equation (15) is solved with the boundary conditions (16)-(19) using the numerical methods introduced in Sec. IV B.

\section{Results}

Figure 5 shows some representative examples of the velocity field $u^{*}\left(x^{*}, y^{*}\right)$ for $M=0, M=1$ and $M=\infty$ for the case of full-slip at the film edge with film thickness $F=0$. From the system of reduced PDEs (15)-(19) it is evident that $Q^{*}$ is a function of the mobility $M$ only (which contains the width of the rivulet since $R=W / 2$ ). The resulting curve is shown in a semilog-plot in Fig. 6. $Q^{*}(M)$ changes steeply around $M$ $\approx 1$, going from $Q^{*}=2.73 \times 10^{-2}$ for low mobilities to $Q^{*}$ $=3.65 \times 10^{-2}$ for high mobilities.

For comparison, the dashed line in Fig. 6 shows the result of simulations with a no-slip condition at the film edge $\left(\partial \Omega_{l f}\right)$. Analytical approximations for this case have been provided by Koehler et al. ${ }^{8}$ We use their expression

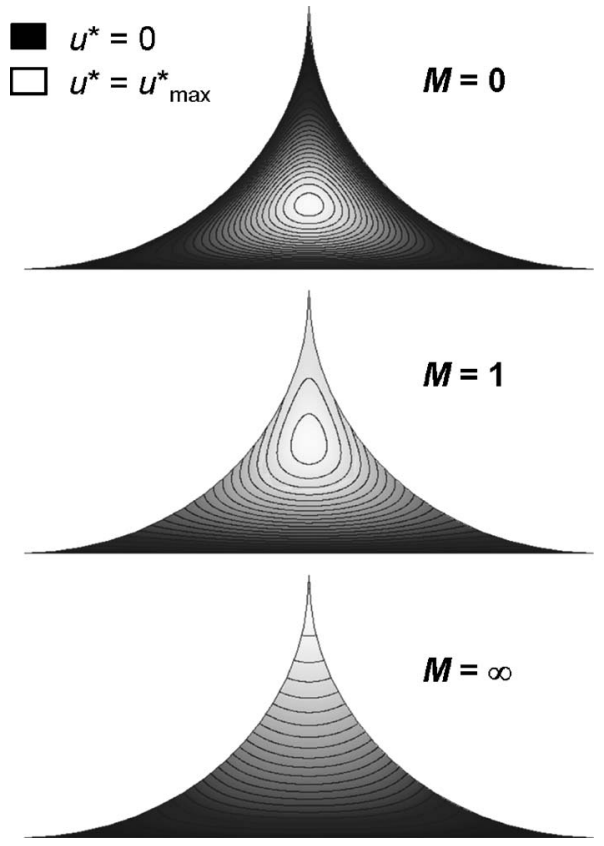

FIG. 5. Examples of flow fields $u^{*}(x, y)$ in a surface Plateau border with zero film width $(F=0)$ for three different mobilities $M$, employing a full-slip condition at the top corner where the film would be attached.

$$
Q^{*}(M) \approx \alpha[\sqrt{M} \operatorname{atan}(\beta \sqrt{M}+\delta)] \sqrt{\operatorname{atan}\left(\frac{\epsilon}{M}\right)},
$$

to obtain an ad hoc fit to our case with full-slip at the film and $F=0$, using $\alpha, \beta, \delta$ and $\epsilon$ as fitting parameters. This is shown as a solid line in Fig. 6 for $\alpha=1.09 \times 10^{-2} ; \beta=9.23$ $\times 10^{-2}, \delta=2.85 \times 10^{-2}$ and $\epsilon=2.582$.

Figure 6 shows that for $F \rightarrow 0$, the boundary condition at the film edge has negligible influence on the overall flow rate in the limits of very mobile or immobile interfaces. For intermediate mobilities $M$, however, the difference between both scenarios is significant, reaching $35 \%$.

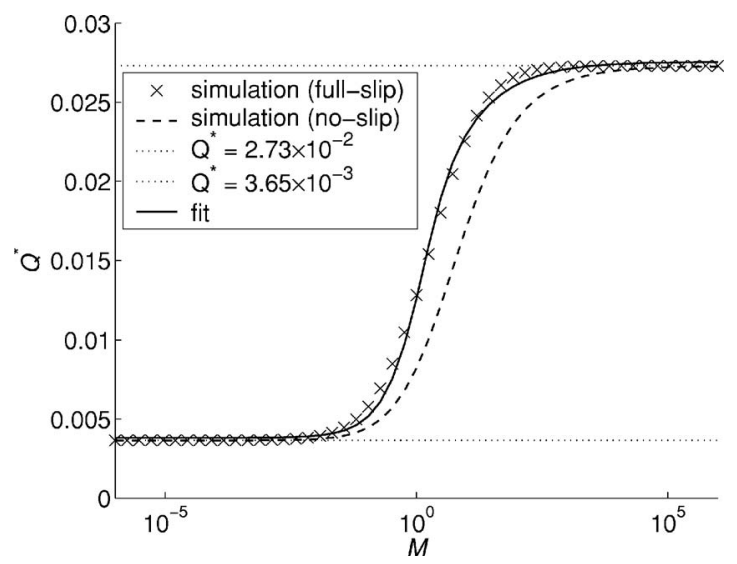

FIG. 6. Reduced flow rate $Q^{*}$ vs mobility $M$ through two surface Plateau borders with zero film width $(F=0)$. The computational result for a full-slip condition at the film edge $(X)$ is fitted (solid line) using Eq. (20) $(\alpha=1.09$ $\times 10^{-2}, \beta=9.23 \times 10^{-2}, \delta=2.85 \times 10^{-2}$, and $\left.\epsilon=2.582\right)$. The dashed line shows the computational result for a no-slip boundary condition at the film edge. 
To illustrate how this effect depends on the relative film thickness $F / R$, Fig. 7 shows the ratio between the flow rates $Q_{\text {noslip }}^{*}$ and $Q_{\text {slip }}^{*}$ with no-slip and full-slip at the film edge, respectively, for a range of relative film widths $F / R$. Additionally, Fig. 8 provides illustrative examples of the flow field for both cases for three different mobilities $(M=0, M$ $=1$, and $M=\infty)$ for the extreme case of $F / R=0.1$. Experimentally we found that $F / R=O\left(10^{-3}\right)$.

Such calculations assume that the film thickness $F$ and the Plateau border size $R$ scale the same way, which is not necessarily the case in experiments. However, it provides an order of magnitude of the influence of the film boundary condition, which we find to be quite significant for intermediate mobilities $M$.

\section{Liquid bridge regime}

With the plate separation $S$ fixed, the gas/liquid interfaces form semicircles of constant radius $R=S / 2$ for all flow rates, while the width $W$ varies. As a result, the rivulet geometry is not self-similar in this regime and the set of PDEs [Eqs. (5), (6), and (8)] cannot be simplified as straightforwardly as in the case of the Plateau border in Sec. IV C. The flow rate $Q$ therefore depends not only on the mobility $M$, but also on the rivulet width $W$.

In order to maintain consistency throughout the article, we use the same reduced variables as for the Plateau border [given in (13), (14), (3), and (18)]. The reduced width $W^{*}$ of the liquid bridge is then given by $W^{*}=W / R=2 W / S$. The plate spacing reduces to $S^{*}=S / R=2$.

Figure 9 shows examples of velocity distributions for two different reduced widths $W^{*}\left(W^{*}=2.4\right.$ and $\left.W^{*}=2.8\right)$ and three different mobilities $M(M=0, M=1$, and $M=\infty)$.

The surface plot in Fig. 10 shows how the reduced flow rate $Q^{*}$ varies with $M$ and $W^{*}$. For clarity, we plot cross sections of this surface in Fig. $11\left(Q^{*}\right.$ vs $\left.W^{*}\right)$ and Fig. $12\left(Q^{*}\right.$ vs $M$ ).

For sufficiently large $W^{*}$, the influence of the gas/liquid boundary becomes a negligible end-effect, and $Q^{*}$ varies linearly with a slope of $2 / 3 W^{*}$ for all mobilities $M$. This computational result can be confirmed analytically by considering a simplified bridge with rectangular cross section and no additional stresses applied on the gas/liquid interfaces. The flow profile between the plates is then of the Poiseuille-type

$$
u^{*}\left(y^{*}\right)=\frac{1}{2}\left(1-y^{* 2}\right),
$$

with translational symmetry along the $x$-direction. In this approximation,

$$
Q^{*}=\frac{1}{4} W^{*} \int_{-1}^{1} u^{*}\left(y^{*}\right) d y^{*}=\frac{2}{3} W^{*} .
$$

\section{EXPERIMENTS}

\section{A. Experimental methods \\ 1. Setup}

The setup is sketched in Fig. 1. It consists of two narrowly spaced float glass plates $(30 \times 20 \mathrm{~cm})$, which guarantee surface smoothness and perfect wetting conditions. Three

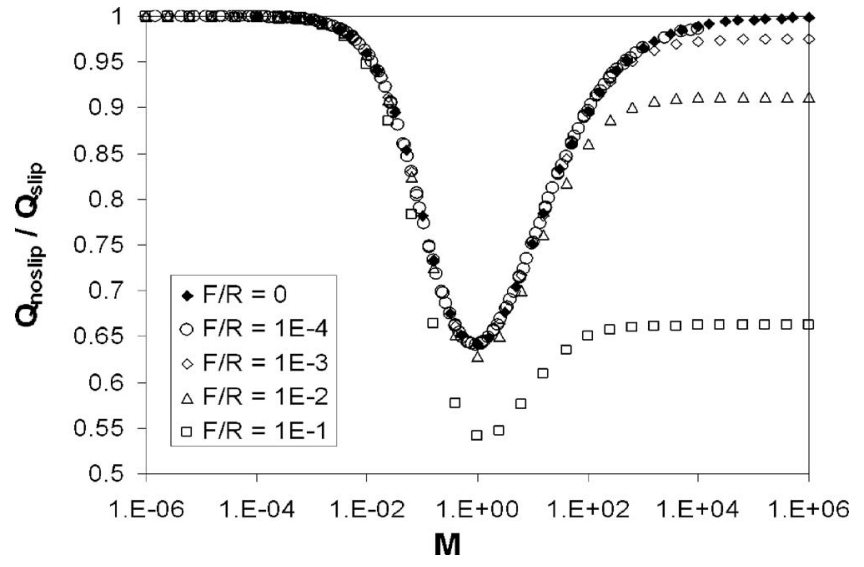

FIG. 7. Ratio of flow rates $Q_{\text {noslip }}^{*}$ and $Q_{\text {slip }}^{*}$ vs the mobility $M$, employing a no-slip and full-slip condition at the film, respectively, for different relative film widths $F / R$.

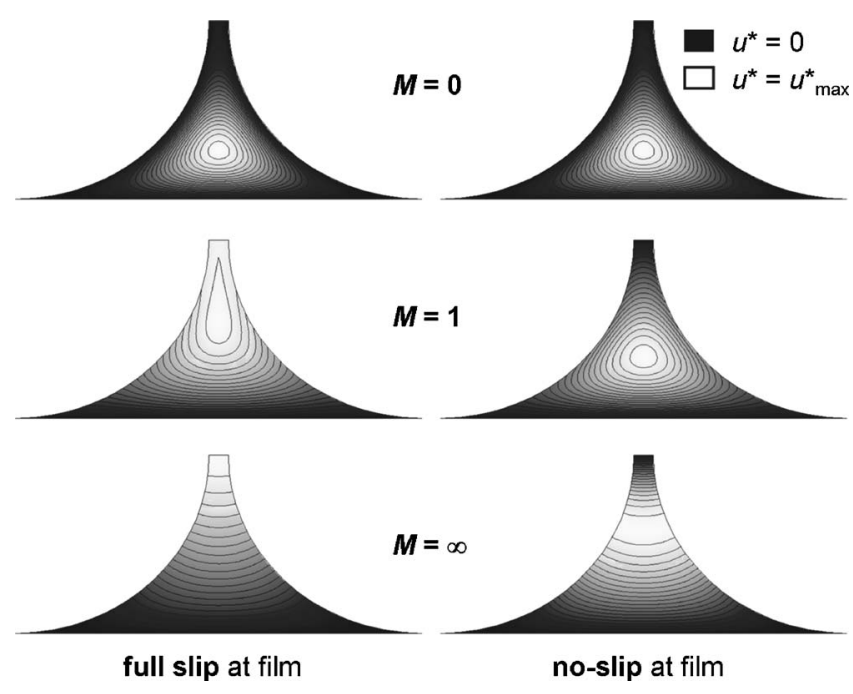

FIG. 8. Examples of flow fields $u^{*}(x, y)$ for three different mobilities $M$ and a large film thickness $F / R=0.1$ to illustrate the influence of a full-slip (left column) and no-slip (right column) boundary condition at the film edge $\partial \Omega_{l f}$.

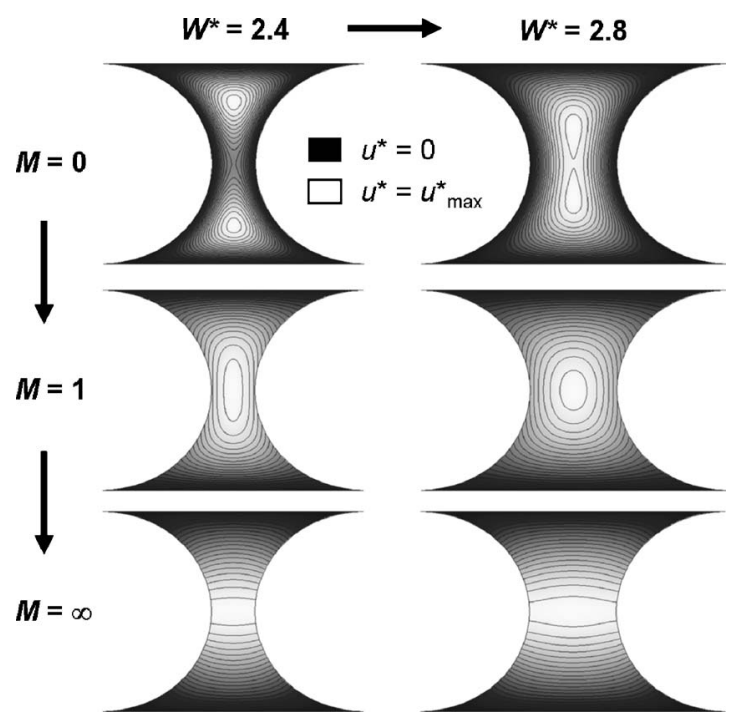

FIG. 9. Examples of velocity fields $u^{*}\left(x^{*}, y^{*}\right)$ for three different mobilities $M$ and two different widths $W^{*}=W / R$. 


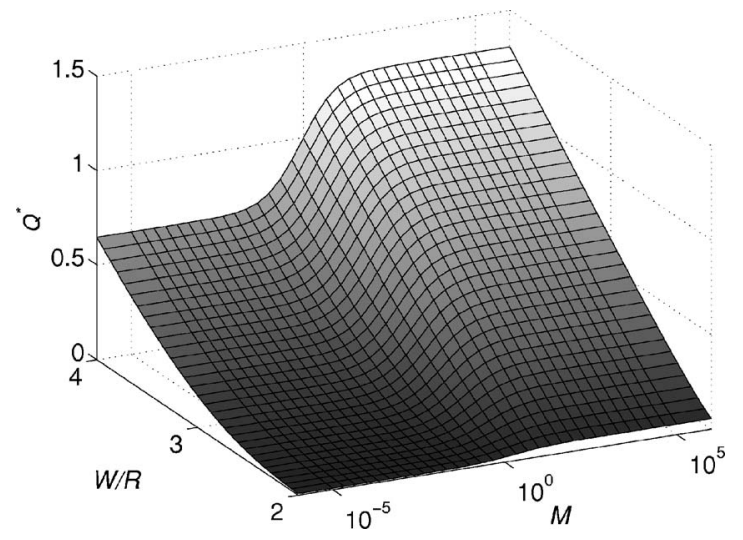

FIG. 10. Surface plot to illustrate how the reduced flow rate $Q^{*}$ through the liquid bridge depends on its mobility $M$ and reduced width $W^{*}=W / R$.

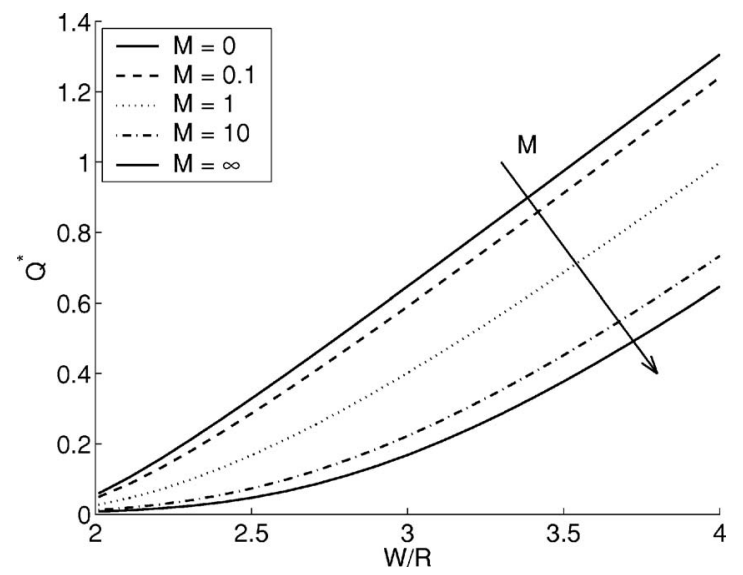

FIG. 11. Cross sections of the surface plot in Fig. 10, showing the variation of the reduced flow rate $Q^{*}$ with the reduced width $W^{*}=W / R$ for five different mobilities $M$. For large $W^{*}$ these tend toward straight lines with slope $2 / 3$, as given by Eq. (22).

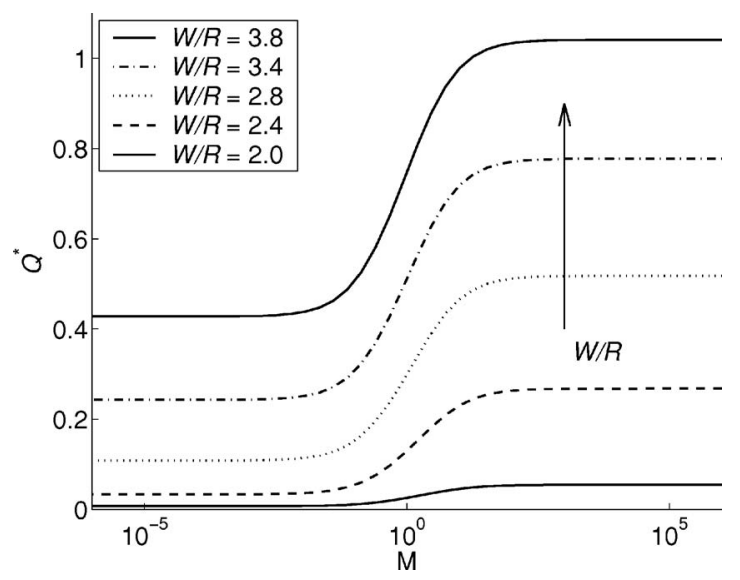

FIG. 12. Cross sections of the surface plot in Fig. 10, showing the variation of the reduced flow rate $Q^{*}$ with the mobility $M$ for five different reduced widths $W^{*}=W / R$. This variation would not occur within a single experiment, since $M$ is constant in the liquid bridge regime, but could be realized by changing $\mu_{s}$.

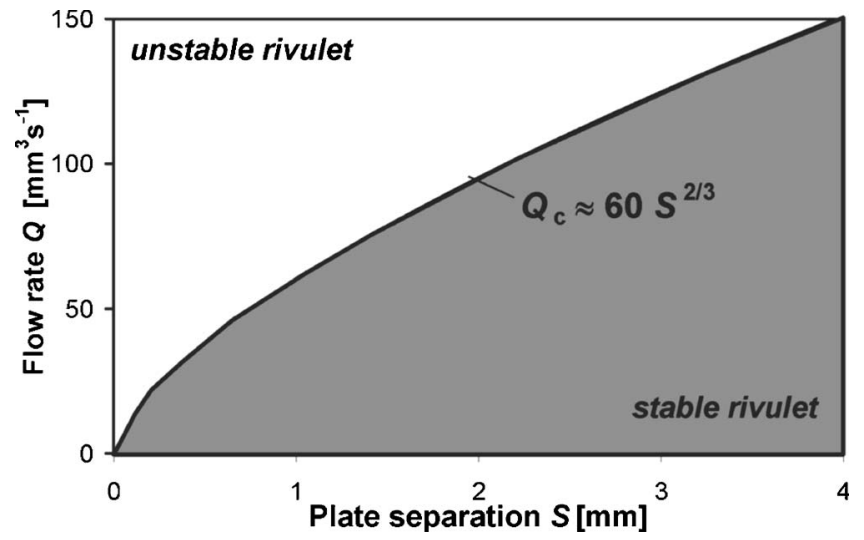

FIG. 13. The rivulet is unstable above a critical flow rate, which depends on the plate separation $S$. Shown here is the experimentally established stability diagram for commercial Fairy Liquid solutions [Eq. (23), Ref. 1]. All our experiments are conducted in the stable (gray) zone.

micrometer screws in a triangular arrangement allow us to control the plate separation $S$ with an accuracy of $0.05 \mathrm{~mm}$. The plates are placed between a diffusive light source (height $40 \mathrm{~cm}$, width $20 \mathrm{~cm}$ ) and a CCD camera. The light source is located $2 \mathrm{~m}$ away from the plates for imaging reasons (Sec. V A 2).

Surfactant solutions of various compositions (Sec. V A 3) are injected at the top of the plates at constant flow rate $Q\left(0.2 \mathrm{~mm}^{3} / \mathrm{s}<Q<100 \mathrm{~mm}^{3} / \mathrm{s}\right)$, which is measured with a high precision-flowmeter.

\section{Measurement of the rivulet width}

The values of the rivulet width $W$, which we present here, are the average of measurements taken at ten different vertical positions on the rivulet at constant flow rate, sufficiently far away $(\geq 5 \mathrm{~cm})$ from the injection point to ensure that the flow is in equilibrium. All measurements were taken before the onset of the wave instability, which occurs at a critical flow rate $Q_{c}(S) .{ }^{1}$ The authors of Ref. 1 established experimentally for solutions of the commercial dishwashing surfactant Fairy Liquid that

$$
Q_{c}=\alpha S^{2 / 3},
$$

with $\alpha \approx 60 \mathrm{~mm}^{7 / 3} \mathrm{~s}^{-1}$. This corresponds to a phase diagram as displayed in Fig. 13, which is consistent with what we found here.

Typical rivulet images are shown in Fig. 14 for different flow rates. The two left images correspond to the Plateau border regime (left-hand side of Fig. 2). The two right images correspond to the liquid bridge regime (right-hand side of Fig. 2). The images are calibrated with an accuracy of about $50 \mu \mathrm{m}$.

As analyzed in detail in Ref. 13, the black stripes are formed as a result of refraction/reflection of light at the boundaries of the rivulet, which acts like an intricately shaped lens. The width of the stripes therefore depends significantly on the lighting conditions. Most importantly, their outer boundaries do not correspond to the actual boundaries of the rivulet. In order to relate these two quantities, we employed computational ray-tracing techniques, ${ }^{13}$ using 


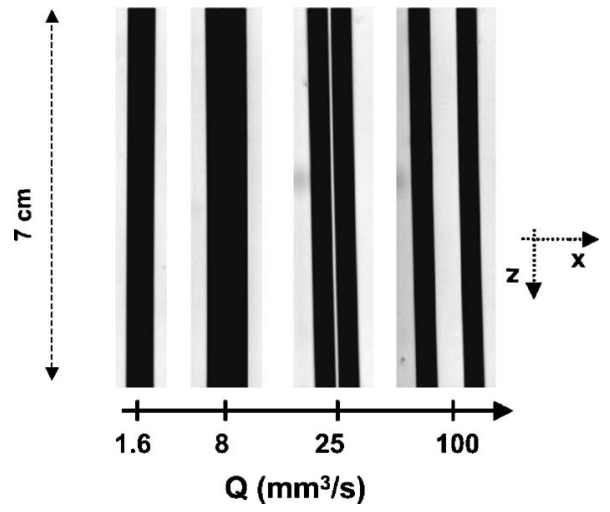

FIG. 14. Typical images of the rivulet for increasing flow rate $Q$. The two left images correspond to the Plateau border regime (left-hand side of Fig. 2 ), while the two right images are in the liquid bridge regime (right-hand side of Fig. 2).

commercial rendering software (3D Studio Max with Final Render) and our own MATLAB ray-tracing programs. In both, the image of the rivulet is predicted for specific lighting and imaging conditions by tracing light rays from the light source through the rivulet to the imaging camera, taking into account the laws of reflection and refraction at the gas/liquid and glass/liquid interfaces.

We find that for our particular setup

$$
W_{\text {actual }}=1.064 W_{\text {apparent }} \text {. }
$$

The experimental results presented in this article have been corrected using this value.

\section{Characterization of the surfactant solutions}

We have used a range of aqueous surfactant and protein solutions with different interfacial and bulk rheological properties. Extra pure Millipore water was used for all solutions.

a. Variation of surface properties. Table I lists the surfactant and protein solutions which we used to obtain systems with different surface viscosities $\mu_{s}$. The main surfactant is the anionic sodium dodecyl sulfate (SDS) at a fixed concentration of $c=5.6 \mathrm{~g} / 1$, which is twice the critical micellar concentration (CMC). To this base solution we add dodecanol (DOH) at various concentrations to increase the interfacial viscosity $\mu_{s}$. We use the milk protein casein for very rigid interfaces. A solution with the commercial dishwashing

TABLE I. Overview of the properties of surfactant and protein solutions used in the experiments, including a mobility value $M$ for the example of $R=0.5 \mathrm{~mm}$. Here, SDS is sodium dodecyl sulfate; DOH is dodecanol, $c$ is the concentration of the surface active agent or protein; $\gamma$ is surface tension; $\mu_{s}$ is the surface shear viscosity; $M$ is the mobility parameter [Eq. (3)].

\begin{tabular}{lcccc}
\hline \hline Surfactant & $\begin{array}{c}c \\
\left(\mathrm{~g} \mathrm{l}^{-1}\right)\end{array}$ & $\begin{array}{c}\gamma \\
\left(\mathrm{N} \mathrm{m}^{-1}\right)\end{array}$ & $\begin{array}{c}\mu_{s} \\
\left(\mathrm{~kg} \mathrm{~s}^{-1}\right)\end{array}$ & $\begin{array}{c}M \text { for } \\
R=0.5 \mathrm{~mm}\end{array}$ \\
\hline SDS & 5.6 & 0.036 & $6.0 \times 10^{-8} \pm 4.0 \times 10^{-8}$ & 300 \\
SDS/DOH & $5.6 / 0.1$ & 0.030 & $1.9 \times 10^{-7} \pm 0.5 \times 10^{-7}$ & 9.5 \\
SDS/DOH & $5.6 / 0.3$ & 0.030 & $2.0 \times 10^{-6} \pm 0.4 \times 10^{-6}$ & 1 \\
"Fairy" & 4 & 0.031 & $1.9 \times 10^{-7} \pm 0.5 \times 10^{-7}$ & 9.5 \\
Casein & 1.5 & 0.041 & $2.5 \times 10^{-6} \pm 0.2 \times 10^{-6}$ & 1.5 \\
\hline
\end{tabular}

TABLE II. Summary of solutions used to investigate the effect of varying the bulk viscosity $\mu_{l}$ (SDS, sodium dodecyl sulfate).

\begin{tabular}{lccc}
\hline \hline Solution & $\mu_{l}\left(10^{-3} \mathrm{Pas}\right)$ & $\gamma\left(10^{-3} \mathrm{~N} \mathrm{~m}^{-1}\right)$ & $\rho\left(\mathrm{kg} \mathrm{m}^{-3}\right)$ \\
\hline SDS +5\% glycerol & 1.1 & 29.0 & 1026 \\
SDS +15\% glycerol & 1.5 & 29.0 & 1052 \\
SDS +25\% glycerol & 2.1 & 30.6 & 1078 \\
SDS +40\% glycerol & 3.6 & 30.5 & 1118 \\
\hline \hline
\end{tabular}

Fairy Liquid serves for comparison with previous work on rivulet flow. ${ }^{1}$ The bulk properties of all solutions (density, bulk viscosity) remained unchanged, i.e., that of water.

We also conducted experiments using a cationic surfactant (tetradecyl trimethyl ammonium bromide, TTAB) to investigate possible differences with the anionic SDS solutions. The results were the same as for SDS solutions within the experimental error and are therefore not reported here.

We determined the surface tension $\gamma$ and surface shear viscosity $\mu_{s}$ for all solutions using a pendant drop technique (ITConcept) and a commercial surface shear rheometer (Sinterface), respectively. In the latter, a metallic cylinder is held vertically by a thin wire and put in contact with the surface of the solution. The cylinder is rotated slightly around the vertical axis such that the surface of the solution is sheared. When the force on the ring is removed it performs damped oscillations from which the shear viscosity can be deduced.

The surface properties are summarized in Table I, in which we also give a representative value of the mobility $M$ for the case of $R=0.5 \mathrm{~mm}$. While the surface tension $\gamma$ varies little, the surface shear viscosity $\mu_{s}$ varies significantly. Accurate measurements of the latter are still quite a challenge, ${ }^{14}$ especially for solutions of low molecular weight surfactants (such as SDS), which tend to be at the limit of the resolution of the measuring device. Also, the coupling with viscous effects in the bulk can be significant, which tends to complicate the interpretation of the data.

The surface shear viscosities measured by us are consistent with those given in the literature, established by different measuring techniques. ${ }^{6,14,15}$ The protein layers (casein) and SDS/DOH interfaces have significantly higher shear viscosities than pure SDS interfaces, as has already been shown in foam drainage experiments. ${ }^{15,16}$

b. Variation of bulk properties. The bulk properties of the liquid are varied by adding glycerol to a pure SDS solution, as listed in Table II. This slightly changes the surface tension $\gamma$ and density $\rho$, but has negligible influence on the surface shear viscosity $\mu_{s}$. The overall behavior of the solution remains Newtonian.

\section{B. Results}

\section{Influence of the surface shear viscosity}

Figure 15 shows the relationship between the flow rate $Q$ and the rivulet width $W$ measured for solutions of different surface viscosity $\mu_{s}$ (as summarized in Table $\mathrm{I}$ in Sec. V A 3), for a plate separation of $S=1 \mathrm{~mm}$. For clarity, the data sets are plotted in two separate graphs. Also shown are the computationally established limits for perfectly immobile 


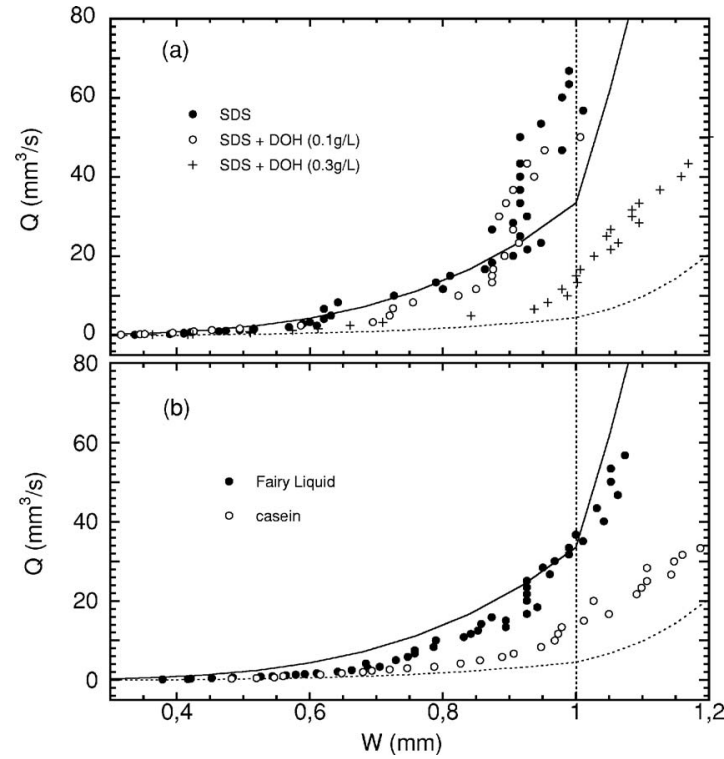

FIG. 15. Flow rate $Q$ as a function of the rivulet width $W$ for different solutions of surface active species (Table I): (a) SDS with DOH, (b) Fairy Liquid and casein. All solutions have the same bulk viscosity $\mu_{l}=1 \mathrm{mPa}$. The dashed and solid line correspond to the computationally established limits for perfectly immobile $\left(\mu_{s}=\infty\right.$ or $\left.M=0\right)$ and perfectly mobile $\left(\mu_{s}=0\right.$, or $M=\infty$ ) gas/liquid interfaces, respectively (Fig. 6 in Sec. IV C and Fig. 11 in Sec. IV D).

$\left(\mu_{s}=\infty\right.$ or $\left.M=0\right)$ and perfectly mobile $\left(\mu_{s}=0\right.$ or $\left.M=\infty\right)$ interfaces from Sec. IV C (Fig. 6) and Sec. IV D (Fig. 11), respectively. They have been redimensionalized using Eq. (18) and the appropriate physical parameters, $\rho$ $=1000 \mathrm{~km} \mathrm{~m}^{-3} ; \mu_{l}=0.001 \mathrm{~Pa} \mathrm{~s} ; g=9.81 \mathrm{~m} \mathrm{~s}^{-2}$.

The general trend for all surfactant solutions is correct. With increasing interfacial viscosity $\mu_{s}$, the same flow rate $Q$ leads to an increased rivulet width $W$, with the casein solution being closest to the rigid interface limit. The Plateau border and liquid bridge regime (Fig. 2) are clearly distinguishable in the case of low-mobility surfactants, in which a pronounced kink is observed in the data around $W=S$. This kink corresponds to the point at which the Plateau borders merge. For low molecular weight surfactants (and low interfacial viscosities) we observe it consistently at $W=0.9$. This could indicate a systematic deviation from our model, for instance regarding the negligible role of stresses exerted by the thin film. However, the apparent discrepancy is close to the limit of accuracy of the measurements. We therefore refrain here from drawing quantitative conclusions.

The role of the interfacial viscosity is particularly well reflected in the variation of the slope in the liquid bridge regime $(W>S=1 \mathrm{~mm})$. In the Plateau border regime all data are very well described by Eq. (20) and we do not find any unexpected behavior as reported by Pitois et al., ${ }^{17}$ who observed flow-rate-dependent surface viscosities.

The Fairy Liquid solution is confirmed to have high values of surface shear viscosity. This is in agreement with previous conclusions from foam drainage experiments. ${ }^{18}$

Lastly, note that similar results are found when the cationic surfactant TTAB is used (with or without dodecanol

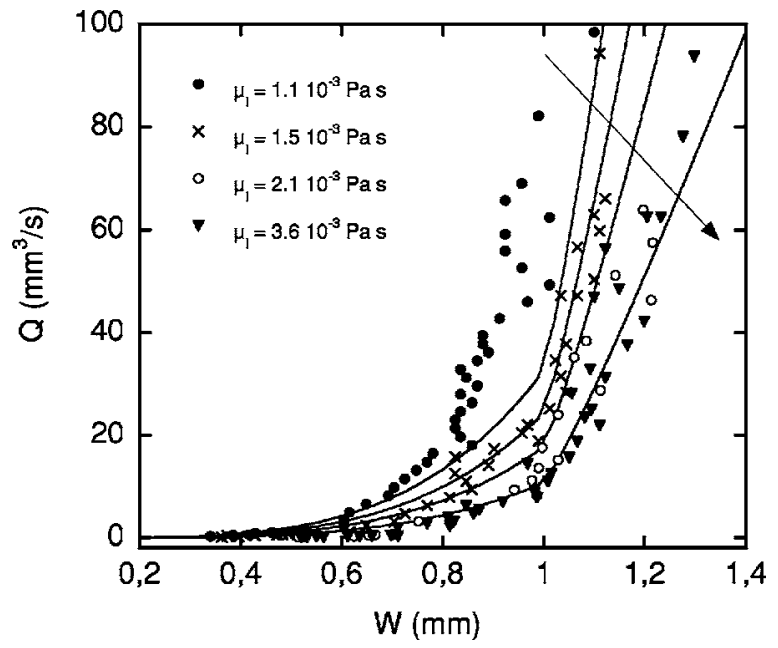

FIG. 16. Flow rate $Q$ as a function of rivulet width $W$ for solutions of different bulk viscosities (with constant interfacial properties). The solid lines are the computational results for each case $\left(\mu_{s}=6.0 \times 10^{-8} \mathrm{~kg} \mathrm{~s}^{-1}\right)$.

added), and that experiments at different separation distances $S(S=0.5,1.2$, and $2 \mathrm{~mm})$ also give similar results.

\section{Results for various bulk properties}

The experimental results for different bulk viscosities $\mu_{l}$ (Table II in Sec. V A 3) are presented in Fig. 16. For each set of data we also show the accompanying computational results (solid lines in Fig. 16) obtained from redimensionalizing Eq. (18) with the appropriate physical parameters, $\rho$ $=1000 \mathrm{~km} \mathrm{~m}^{3} ; \mu_{s}=6.0 \times 10^{-8} \mathrm{~kg} \mathrm{~s}^{-1} ; g=9.81 \mathrm{~m} \mathrm{~s}^{-2}$. As in the previous section, the data are qualitatively in agreement with the simulations and both regimes (Fig. 2) are clearly separated by a kink around $W=S$.

The addition of $5 \%$ of glycerol only slightly increases the viscosity (Table II). It is thus consistent to find a behavior similar to the one of the pure SDS solution, as shown in Fig. 15 (with the same localization of the kink for $W$ just below $1 \mathrm{~mm})$. For the other glycerol concentrations we find that the experimental curves remain always close to the simulations.

\section{CONCLUSIONS AND OUTLOOK}

We have presented computational and experimental investigations into stable rivulet flow between plates, concentrating in particular on the influence of the interfacial and bulk shear viscosity of the surfactant solutions. In both cases, modelling results show good agreement with experiments.

The order of magnitude of the interfacial shear viscosities which we find by comparing the experimental results and computational predictions agrees with those independently determined with a surface rheometer (Sec. V A 3) or reported in the literature. ${ }^{6,14} \mathrm{We}$ also find that the data are generally better described by assuming full slip at the film edge $\partial \Omega_{l f}$ (Fig. 4).

In order to draw proper quantitative conclusions, it will be essential to significantly improve the accuracy of our simple experimental setup. We are optimistic that this can be achieved by improving the control of the plate spacing and the flow rate, and the measurement of the rivulet width. If 
this can be done to sufficient accuracy, such an apparatus could provide an alternative and straightforward measurement technique to determine interfacial shear viscosities of solutions of surface active species.

Our computational model provides detailed information on the relationship between flow profiles, rivulet geometry, and interfacial properties of the stable rivulet and may therefore contribute to investigations into the destabilizing and stabilizing mechanisms at work in rivulet meanders. ${ }^{1}$ Considering the maximum velocities predicted in Appendix B, one finds that for a rivulet with $R \approx 10^{-3}$, Reynolds numbers can go up to 5000. We have not observed turbulent flow in our experiments, but it might play a role for the stability of the rivulet. ${ }^{1}$ Additionally, for surface active species with adsorption time scales of the order of typical inverse shear rates encountered in the flow, one might need to take into account a variation of surface tension. This could lead to a change of shape of the rivulet cross section and other dynamic effects. For the solutions investigated here we do not expect this to be the case.

In more general terms, the rivulet system (mostly in the Plateau border regime) provides further indication of the role of the boundary conditions on flow through Plateau borders in foam drainage. ${ }^{18}$ The coupling between the flow resistance and the interfacial mobility of these elegant channels has received increasing attention in recent years. ${ }^{5,8,19,20}$ Our results support previous investigations. Additionally they show that the role of the transition zone between the Plateau border and films deserves more careful consideration, which might be the heart of current limits to theoretical descriptions of foam drainage for small bubbles or large flow rates. ${ }^{3,9}$

Answers to such questions may also provide insight into mechanisms at work in instabilities in foams, especially at the onset of convective motion in forced drainage. ${ }^{21}$ For instance, what happens when two Plateau borders merge to form a liquid bridge? Some of our high mobility data indicate a Plateau in the $W(Q)$ relationship at this point, which may be a result of the change in boundary conditions. Such information may prove important in understanding the physics of topological rearrangements in foams. ${ }^{22}$

In comparison to the flow conditions encountered in a typical bulk foam (with bubble diameters of the order of millimeters and less), those accessed in our investigations have been rather extreme. First, the rivulet represents channels which form where foams are in contact with container walls. These not only differ from interior Plateau borders in geometry but are also subject to different boundary conditions; the solid wall provides a large source of dissipation, which limits the maximum possible flow velocities. More importantly, the typical length scale in our experiment was $R \approx 1 \mathrm{~mm}$, which is an order of magnitude larger than in many common liquid foams. As a result, fluid velocities and mobilities encountered in our experiments are significantly larger. Large values of $R$ also lead to low capillary pressures in the Plateau border $\left(P_{c} \sim \gamma / R \leq 10 \mathrm{~Pa}\right)$, implying practically no suction from the Plateau borders on the film. Last but not least, the film aspect ratio in the rivulet is very different from those in typical foams and there are no horizontal boundaries, which could limit the flow and induce circula- tion. Despite (or because of) these differences, we believe that important lessons can be learned by comparing rivulet and foam flow. Future experiments with increased accuracy will be able to access length scales and flow parameters more comparable to those encountered in common liquid foams.

An understanding of rivulet flow may also provide valuable insights into the role of pressure driven drainage in foams, which is encountered in recent developments in discrete microfluidics. ${ }^{23,24}$ In this, monolayers of bubbles are pushed through narrow channels, thereby forming the same kind of channel cross sections as analyzed here. Large dissipation leads to high pressure gradients, which drives the liquid through these channels, which in turn may have significant influence on a number of physical and physicochemical properties of these systems.

In terms of the simulations we may consider this study as further indication of the general success of the application of finite element methods to solve fluid dynamic problems in foams, i.e., problems with complex geometrical and physical boundary conditions. ${ }^{8,25}$ For easier comparison between theory and experiment it will be desirable to establish analytical approximations in the style of Koehler et al. ${ }^{8}$ and Nguyen, ${ }^{5}$ who have provided relationships for interior Plateau borders and surface Plateau borders with a no-slip condition at the film. We hope that the information provided here will encourage attempts to provide similar approximations for the case of surface Plateau borders with more flexible boundary condition at the film and also for liquid bridges.

\section{ACKNOWLEDGMENTS}

The authors acknowledge funding from the European Space Agency (Contract Nos. MAP AO-99-108:C14914/02/ NL/SH and MAP AO-99-075:C14308/00/NL/SH), Enterprise Ireland (Contract No. BRG SC/2002/011), and the Science Foundation of Ireland (Contract No. RFP 05/RFP/ PHY0016). W.D. was an IRCSET Postdoctoral Fellow, funded by Enterprise Ireland.

\section{APPENDIX A: IMPLEMENTATION IN COMSOL}

Following from Sec. IV we implement the following in COMSOL:

- Subdomain $\Omega$ weak equations:

$$
\begin{aligned}
& \text { “-nl*ux*ux_test-nl *uy*uy_test } \\
& + \text { rhog*u_test" }
\end{aligned}
$$

- Gas/liquid boundary $\partial \Omega_{l g}$ weak equation:

$$
\begin{aligned}
& \text { “- ns * (uTx *tx +uTy*ty)*(uTx_test*tx } \\
& \text { + uTy_test* ty)“ }
\end{aligned}
$$

Here, "nl," "ns," and "rhog" are $\mu_{l}, \mu_{s}$ and $\rho g$, respectively. The remainder are COMSOL notations for the velocity field " $u$ " and its derivatives, the test function " $u$ _test" and its derivatives, and the components of the tangential unit vector "t." 


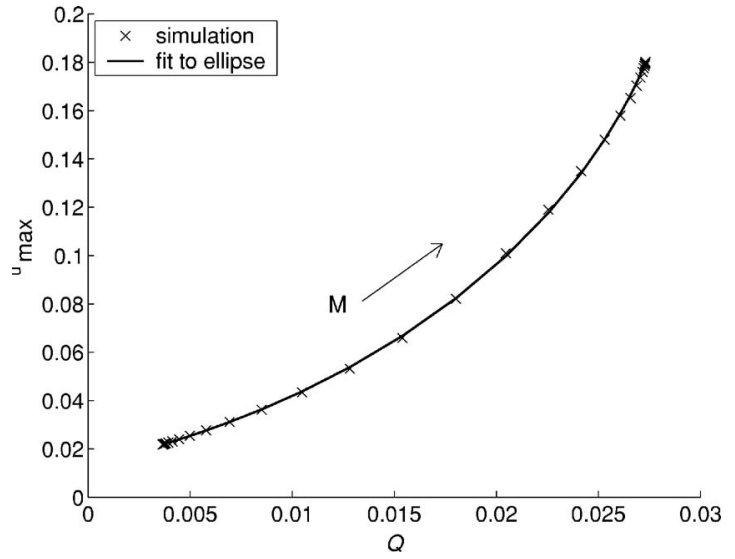

FIG. 17. In the Plateau border regime (Fig. 2), the reduced maximum velocity $u_{\max }$ vs the reduced flow rate $Q$ is fitted very well by an ellipse [Eq. (B1) $\left.u_{0}=0.259, a_{u}=0.254, Q_{0}=-1.056 \times 10^{-2}, b_{Q}=3.984 \times 10^{-2}\right]$.

\section{APPENDIX B: MAXIMUM VELOCITIES}

For many questions regarding the nature of the flow, it is important to know the maximum velocity $u_{\max }$ in the rivulet. Since this quantity is not easily accessible in the experiment, we present here the computational results for both regimes. We find that $u_{\max }$ is related to the flow rate $Q$ by simple relationships.

In this section, all quantities are given in their reduced form. For clarity we omit the superscript $*$ in the text.

\section{Surface Plateau border regime}

For each mobility $M$ one obtains a unique $\left(u_{\max }, Q\right)$-couple. These are presented in Fig. 17 for the whole range of mobilities $M$ for the case of zero film width $(F=0)$ and full-slip at the film edge. As can be seen in Fig. 5, the position of the maximum velocity moves from the center to the corner point of the Plateau border as the mobility $M$ increases.

For reasons not yet clear to us, the curve $u_{\max }(Q)$ is very well described by an ellipse

$$
\left(\frac{u_{\max }-u_{0}}{a_{u}}\right)^{2}+\left(\frac{Q-Q_{0}}{b_{Q}}\right)^{2}=1,
$$

which is shown by the solid line in Fig. 17. $\left(u_{0}=0.259, a_{u}\right.$ $=0.254, Q_{0}=-1.056 \times 10^{-2}, b_{Q}=3.984 \times 10^{-2}$.)

\section{Liquid bridge regime}

In this regime, the position and magnitude of the maximum velocity $u_{\max }$ depend on the mobility $M$ and the width $W$ of the liquid bridge. We therefore recover a set of curves for different mobilities, of which three representative examples $(M=0, M=10, M=\infty)$ are shown in Fig. 18. They are very well fitted by exponentials

$$
U_{\max }(Q)=0.5-\alpha \exp \left\{-\beta Q^{\delta}\right\},
$$

using $\alpha, \beta$ and $\delta$ as fitting parameters. The results are shown by the solid lines in Fig. 18; the corresponding fitting parameters are provided in Table III.

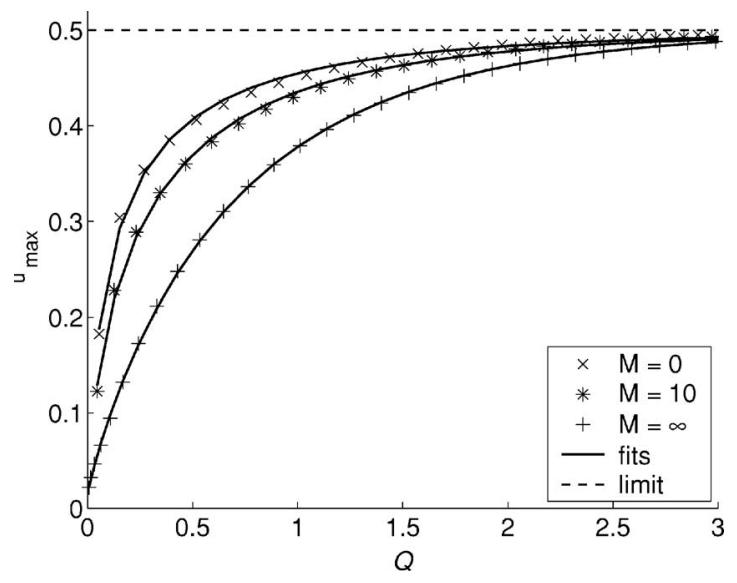

FIG. 18. Reduced maximum velocity $u_{\max }$ vs reduced flow rate $Q$ in a liquid bridge for three different mobilities. All data are very well fitted by Eq. (B2). The asymptotic limit of $u_{\max }=0.5$ is given by setting $y=0$ in Eq. (21).

All curves approach $u_{\max }=0.5$ for large flow rates, which is also found analytically by setting $u_{\max }=u(R)$ in Eq. (21).

The origin of the exponential form of Eq. (B2) may be captured by considering the influence of the gas/liquid boundaries as an exponentially decaying perturbation on the undisturbed flow. A general argument could proceed as follows.

Let us consider liquid bridges which are sufficiently wide, such that their geometry may be approximated by a rectangle of width $W$ (Fig. 19), with straight gas/liquid interfaces rather than arcs of circles (Fig. 2). For tangentially fully mobile gas/liquid interfaces $(M=\infty), u_{\max }=0.5$ is given by Eq. (21). It is the same result as for an infinitely wide bridge, as the gas/liquid interface does not impose additional stresses on the flow. The corresponding velocity profile $u_{\infty}(y)$ has translational symmetry along the $x$-axis. Gas/liquid interfaces with finite mobility $M$ impose a perturbation on this velocity profile, which we denote as $\hat{u}(x, y)$, such that the overall velocity field is given as

$$
u(x, y)=u_{\infty}(y)-\hat{u}(x, y) .
$$

Since the $u(x, y)$ and $u_{\infty}(y)$ satisfy the Poisson equation (5), $\hat{u}(x, y)$ must satisfy the Laplace equation

$$
\Delta \hat{u}(x, y)=0,
$$

because of linearity.

A possible solution to this is given by a Fourier series with terms that exponentially decay along the $x$-direction away from the gas/liquid boundary,

TABLE III. Fitting parameters from fitting Eq. (B2) to $u_{\max }^{*}\left(Q^{*}\right)$ shown in Fig. 18.

\begin{tabular}{llll}
\hline \hline & $M=\infty$ & $M=10$ & \multicolumn{1}{c}{$M=0$} \\
\hline$\alpha$ & 0.48624 & 0.50151 & 0.53376 \\
$\beta$ & 1.3844 & 2.0549 & 2.4713 \\
$\delta$ & 0.8932 & 0.6122 & 0.50804 \\
\hline \hline
\end{tabular}




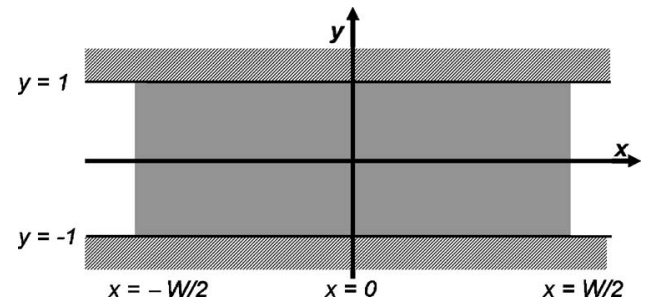

FIG. 19. Rectangular liquid bridge and corresponding coordinates used for approximation (B8).

$$
\begin{aligned}
\hat{u}(x, y)= & \sum_{n} a_{n} \cos \left(\frac{n \pi}{2} y\right)\left(\exp \left\{-\frac{n \pi}{2}\left(x+\frac{W}{2}\right)\right\}\right. \\
& \left.+\exp \left\{\frac{n \pi}{2}\left(x-\frac{W}{2}\right)\right\}\right) .
\end{aligned}
$$

The Fourier coefficients $a_{n}$ are functions of the mobility $M$, i.e., $a_{n}(M)$.

Concentrating on the $n=1$ term of Eq. (B5) as the dominant contribution to the perturbation and locating the maximum velocity at the center point of the rectangle, one obtains

$$
\begin{aligned}
u_{\max }(W, M) & =u_{\infty}(y=0)-\hat{u}(x=0, y=0) \\
& \approx 0.5-2 a_{1}(M) \exp \left\{-\frac{\pi}{4} W\right\} .
\end{aligned}
$$

After integration over the rectangle, this provides a rough estimate for the flow rate

$$
Q(W, M)=\frac{2}{3} W-\frac{8}{\pi} a_{1}(M)\left[1-\exp \left\{-\frac{\pi}{4} W\right\}\right] .
$$

This captures the general features of the $Q(W)$ curves provided in Fig. 11, in particular the mobility-dependant change of slope for small $W$ and the constant slope for large $W$.

Using $Q \propto \frac{2}{3} W$ as a first approximation for highly mobile gas/liquid interfaces $(M \approx \infty)$ one therefore obtains

$$
u_{\max }(Q, M) \approx 0.5-2 a_{1}(M) \exp \left\{-\frac{3 \pi}{8} Q\right\},
$$

which is reasonably close to the fit given in Fig. 18 and Table III.

\footnotetext{
${ }^{1}$ W. Drenckhan, S. Gatz, and D. Weaire, "Wave patterns of a rivulet of surfactant solution in a Hele-Shaw cell," Phys. Fluids 16, 3115 (2004).

${ }^{2}$ D. A. Edwards, H. Brenner, and D. T. Wasan, Interfacial Transport Pro-
}

cesses and Rheology (Butterworth-Heinemann, Stonheam, 1991).

${ }^{3}$ A. Saint-Jalmes, "Physical chemistry in foam drainage and coarsening," Soft Matter 2, 836 (2006).

${ }^{4}$ R. A. Leonard and R. Lemlich, "A study of interstitial liquid flow in foam," AIChE J. 11, 18 (1965).

${ }^{5}$ A. V. Nguyen, "Liquid drainage in single plateau borders in foams," J. Colloid Interface Sci. 249, 194 (2002).

${ }^{6}$ S. A. Koehler, S. Hilgenfeldt, E. R. Weeks, and H. A. Stone, "Drainage of single plateau borders: Direct observation of rigid and mobile interfaces," Phys. Rev. E 66, 040601 (2002).

${ }^{7}$ M. Durand and D. Langevin, "Physicochemical approach to the theory of foam drainage," Eur. Phys. J. E 7, 35 (2002).

${ }^{8}$ S. A. Koehler, S. Hilgenfeldt, and H. A. Stone, "Foam drainage on the microscale. I. Modeling flow through single plateau borders," J. Colloid Interface Sci. 276, 420 (2004).

${ }^{9}$ S. A. Koehler, S. Hilgenfeldt, E. R. Weeks, and H. A. Stone, "Foam drainage on the microscale. II. Imaging flow through single plateau borders," J. Colloid Interface Sci. 276, 439 (2004).

${ }^{10}$ D. Exerova and P. M. Kruglyakov, Foam and Foam Films; Theory, Experiment, Application (Elsevier Science, Amsterdam, 1998), Vol. 5.

${ }^{11}$ C. Marangoni, "Über die Ausbreitung der Tropfen einer Flüssigkeit auf der Öberfläche einer anderen," Ann. Phys. 143, 337 (1871).

${ }^{12}$ R. L. Taylor and O. C. Zienkiewicz, The Finite Element Method (Butterworth-Heinemann, Oxford, 2000), Vols. 1-3.

${ }^{13}$ A. van der Net, L. Blondel, A. Saugey, and W. Drenckhan, "Simulating and interpreting images of foams with computational ray-tracing techniques," Colloids Surf., A 309, 159 (2007).

${ }^{14} \mathrm{P}$. Stevenson, "Remarks on the shear viscosity of surfaces stabilized with soluble surfactants," J. Colloid Interface Sci. 290, 603 (2005).

${ }^{15}$ A. Saint-Jalmes, Y. Zhang, and D. Langevin, "Quantitative description of foam drainage: Transitions with surface mobility,” Eur. Phys. J. E 15, 53 (2004).

${ }^{16}$ M. Durand, G. Martinoty, and D. Langevin, "Liquid flow through aqueous foams: From the plateau border-dominated regime to the node-dominated regime," Phys. Rev. E 60, R6307 (1999).

${ }^{17} \mathrm{O}$. Pitois, C. Fritz, and M. Vignes-Adler, "Hydrodynamic resistance of a single foam channel," Colloids Surf., A 261, 109 (2005).

${ }^{18}$ D. Weaire and S. Hutzler, The Physics of Foams (Clarendon, Oxford, 1999).

${ }^{19}$ O. Pitois, C. Fritz, and M. Vignes-Adler, "Liquid drainage through aqueous foam: study of the flow on the bubble scale," J. Colloid Interface Sci. 282, 458 (2005).

${ }^{20}$ S. J. Cox, D. Weaire, S. Hutzler, J. Murphy, R. Phelan, and G. Verbist, "Applications and generalizations of the foam drainage equation," Proc. R. Soc. London, Ser. A 456, 2441 (2000).

${ }^{21}$ S. Hutzler, D. Weaire, and R. Crawford, "Convective instability in foam drainage," Europhys. Lett. 41, 461 (1998).

${ }^{22}$ M. Durand and H. A. Stone, "Relaxation time of the topological T1 process in a two-dimensional foam," Phys. Rev. Lett. 97, 226101 (2006).

${ }^{23}$ W. Drenckhan, S. J. Cox, G. Delaney, H. Holste, D. Weaire, and N. Kern, "Rheology of ordered foams - on the way to discrete microfluidics," Colloids Surf., A 263, 52 (2005).

${ }^{24}$ J. P. Raven, P. Marmottant, and F. Graner, "Dry microfoams: formation and flow in a confined channel," Eur. Phys. J. B 51, 137 (2006).

${ }^{25}$ A. Saugey, W. Drenckhan, and D. Weaire, "Wall slip of bubbles in foams," Phys. Fluids 18, 053101 (2006). 